\title{
Effects of zoledronic acid and ibandronate in the treatment of cancer pain in rats with lung cancer combined with bone metastases
}

\author{
GENGSHEN WANG ${ }^{1}$, JIUYI CHEN $^{2}$, RUOFEI MA ${ }^{3}$, WEIYUAN XU ${ }^{4}$, CHUNLU YAN $^{5}$ and CUNLIANG NIU ${ }^{1}$ \\ Departments of ${ }^{1}$ Orthopaedics and ${ }^{2}$ Respiratory Medicine, Wuwei People's Hospital, Wuwei, Gansu 733000; \\ ${ }^{3}$ Department of Oncological Surgery, Gansu Provincial Cancer Hospital, Wuwei, Gansu 730050; ${ }^{4}$ Department of \\ Orthopaedics, Lanzhou University Second Hospital, Lanzhou, Gansu 730030; ${ }^{5}$ The Animal Experimental Center, \\ Gansu University of Chinese Medicine, Lanzhou, Gansu 730020, P.R. China
}

Received December 15, 2017; Accepted April 26, 2018

DOI: $10.3892 / \mathrm{ol} .2018 .8804$

\begin{abstract}
The aim of the study was to investigate the efficiency and safety of zoledronic acid and ibandronate in the treatment of rats with lung cancer combined with bone metastases. A total of 124 rats with lung cancer bone metastasis were established. Rats were randomly divided into A, B and C groups $(n=30)$. Rats in group A were treated with ibandronate combined with zoledronic acid, rats in group B were treated with zoledronic acid monotherapy, and rats in group $\mathrm{C}$ were treated with ibandronate monotherapy. Rats in group $\mathrm{A}$ were injected subcutaneously with zoledronic acid $0.1 \mathrm{mg} /$ $\mathrm{kg}$ and ibandronate $10 \mu \mathrm{g} / \mathrm{kg}$, once per week for 12 weeks; rats in group B were injected subcutaneously with zoledronic acid, and rats in group $\mathrm{C}$ were injected subcutaneously with ibandronate, the same method as the treatment group. The remaining $34 \mathrm{SD}$ rats were not treated to serve as the control group. Treatment efficacy and physical improvement in 8 weeks were observed, and improvement of pain behavior in rats was evaluated to reflect the effect of drug treatment. Of the 30 rats in group A, 25 showed different degrees of remission, 5 rats showed no improvement and the effective rate was $83.3 \%$. Of the 30 rats in group B, 21 showed different degrees of remission, 9 rats showed no improvement and the effective rate was $70 \%$. Of the 30 rats in group C, 20 showed different degrees of remission, 10 rats showed no improvement and the effective rate was $66.7 \%$. Statistically significant differences in total effective rate were found among three groups, and the combined method showed the highest effective rate $(\mathrm{P}<0.05)$. Ibandronate combined with zoledronic acid has a good
\end{abstract}

Correspondence to: Dr Jiuyi Chen, Department of Respiratory Medicine, Wuwei People's Hospital, 1 Xuanwu Street, Wuwei, Gansu 733000, P.R. China

E-mail: dreamer1980@126.com

Key words: ibandronate, zoledronic acid, lung cancer bone metastasis, rat model, efficacy therapeutic effect on cancer pain caused by bone metastases from lung cancer.

\section{Introduction}

Lung cancer is a common type of clinical malignant tumor with high incidence and mortality in the respiratory system. Lung cancer seriously affects the health and life of patients $(1,2)$. With the highest incidence among all the subtypes of lung cancer, non-small cell lung cancer is a main cause of death in patients $(3,4)$. Metastasis is common in malignant tumors, and bone metastasis is the most common type, and bone metastases is observed in approximately $20-40 \%$ of patients with lung cancer (5). Therefore, how to improve the treatment of bone metastases in lung cancer patients is the key to improving their quality of life and survival.

At present, treatment with bisphosphonate drugs is an important method in the treatment of bone metastasis from lung cancer $(6)$. Studies have shown that $(7,8)$ bisphosphonates can significantly relieve bone pain and collapse caused by bone metastasis. With direct antitumor effects, bisphosphonates can inhibit the proliferation and diffusion of cancer cells, induce the apoptosis of cancer cells, and inhibit angiogenesis. With direct antitumor activities, zoledronic acid can effectively inhibit the proliferation of small cell lung cancer cells $(9,10)$. Ibandronate has been proved to be effective in the treatment of bone cancer pain and bone collapse caused by bone metastases.

In this study, lung cancer bone metastases rat model was established to explore the therapeutic effect of zoledronic acid and ibandronate. The results are as follows.

\section{Materials and methods}

Subjects. A total of 124 female SD rats were purchased from Experimental Animal Center of Jilin University [certificate number: SCXK (Kyrgyzstan) 2008-0004]. LAD0011 feed was provided by Trophic Animal Feed High-Tech Co., Ltd. (Nantong, China). All the rats were aged 8.5 weeks on average, weighed 200-225 g and raised at indoor temperature of $21.5 \pm 0.5^{\circ} \mathrm{C}$ and humidity of $45-65 \%$ with fluorescent 
Table I. Comparison of basic information of rats.

\begin{tabular}{lccccc}
\hline Parameters & Control & A & B & C & P-value \\
\hline Weight after model construction $(\mathrm{g})$ & $219.8 \pm 10.2$ & $222.4 \pm 11.3$ & $207.6 \pm 12.5$ & $226.1 \pm 10.6$ & 0.785 \\
Weight before model construction $(\mathrm{g})$ & $169.4 \pm 7.3$ & $176.5 \pm 6.8$ & $164.2 \pm 7.5$ & $182.1 \pm 7.2$ & 0.645 \\
Length (cm) & $18.3 \pm 1.2$ & $19.5 \pm 1.1$ & $17.9 \pm 1.3$ & $20.4 \pm 0.9$ & 0.693 \\
Age (week) & $8.1 \pm 0.4$ & $8.5 \pm 0.2$ & $8.4 \pm 0.3$ & $8.6 \pm 0.1$ & 0.899 \\
Glucose (mmol/l) & $82.4 \pm 11.3$ & $79.8 \pm 12.1$ & $86.2 \pm 11.6$ & $84.1 \pm 11.5$ & 0.685 \\
\hline
\end{tabular}

lighting. All rats were allowed to access food and water freely. The study was approved by the Ethics Committee of Wuwei People's Hospital (Wuwei, China).

Rat model establishment. Rat model was constructed according to the methods described by Miller et al (11). Briefly, H1299 cells were cultured with Roswell Park Memorial Institute (RPMI) medium (Invitrogen; Thermo Fisher Scientific, Grand Island, NY, USA) containing $10 \%$ fetal bovine serum in an incubator $\left(37^{\circ} \mathrm{C}, 5 \%\right.$ carbon dioxide). Adjusting concentration, $100 \mu 1\left(1 \times 10^{5}\right)$ was injected into each rat by left ventricular. Rat model established 5 days. In the first $10 \mathrm{~min}$ before model establishment, rats were injected with $10 \mathrm{mg} / \mathrm{kg}$ low molecular weight heparin enoxaparin $(1 \mathrm{mg} / \mathrm{ml})$ through tail vein to prevent thrombosis. After model construction, rats were kept in a laminar flow cage and the activities were monitored.

Treatment. The 124 rats with lung cancer bone metastasis were randomly divided into A, B and C groups ( $\mathrm{n}=30)$. Group A was treated with ibandronate combined with zoledronic acid, group B was treated with zoledronic acid monotherapy, and group $\mathrm{C}$ was treated with ibandronate monotherapy. Rats in group A were injected subcutaneously with zoledronic acid at a dose of $0.1 \mathrm{mg} / \mathrm{kg}$ and ibandronate at a dose of $10 \mu \mathrm{g} / \mathrm{kg}$, once per week for 12 weeks; rats in group B were injected subcutaneously with zoledronic acid, and rats in group $\mathrm{C}$ were injected subcutaneously with ibandronate, the same method as the treatment group. No treatment was performed for the remaining 34 rats, so those rats were used as the control group.

Observation indicators. The $150 \mathrm{SD}$ rats were subjected to radioisotope scanning, and radioisotope accumulation in bone indicated positive bone metastasis. Based on this, 124 rats showed bone metastasis. Changes of body weight were observed. Therapeutic effect was evaluated by comparing the pain behavior of treated rats and control group. Improved pain behavior was defined as effective treatment. Evaluation of physical changes in rats was performed using a modified tail suspension test (TST).

Observation of pain behavior: Paw withdrawal latency (PWL): Rats were transferred to observation cage. When rats became quiet, light was focused on the middle of the toes. The upper limit of PWL was set as $20 \mathrm{sec}$ to avoid burns. The period from the beginning of the irradiation to rats showing lifted legs or escaped was taken as the thermal pain threshold. This experiment was performed 3 times with an interval of $10 \mathrm{~min}$ to calculate the average time. PWL determined at 1 day before model construction was used as the baseline value.

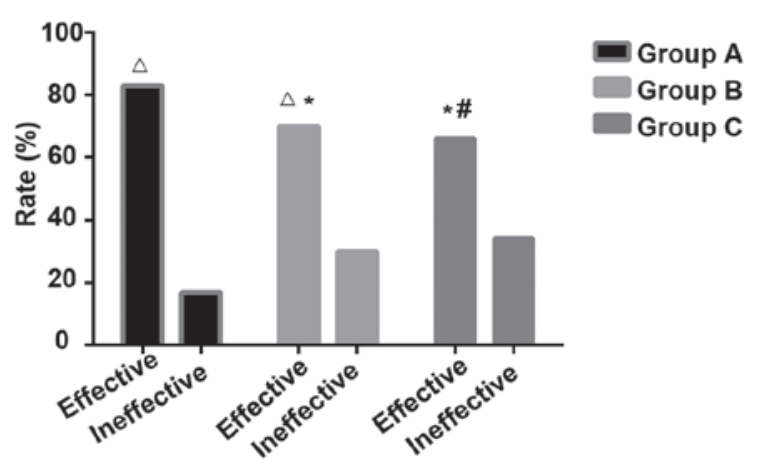

Figure 1. Observation of treatment efficacy of three groups. Cancer pain in 25 out of 30 rats of group A were relieved to varying degrees, and the effective rate was $83.3 \% ; 21$ out of 30 rats of group B were relieved to varying degrees, and the effective rate was $70.0 \%$; 20 out of 30 rats of group $\mathrm{C}$ were relieved to varying degrees, and the effective rate was $66.7 \%$. Group A showed the highest effective rate $\left({ }^{\triangle} \mathrm{P}=0.02\right.$, compared to groups $\mathrm{B}$ and $\left.\mathrm{C}\right)$, but no significant differences in the effective rate were found between group $\mathrm{B}$ and $\mathrm{C}\left({ }^{* \#} \mathrm{P}=0.1432\right)$.

Paw withdrawal mechanical threshold (PWT): The rat foot was stimulated by von Frey filaments with different thresholds, and the upper limited was set as $26 \mathrm{~g}$. Rapid paw withdrawals during or right after stimulation were treated as positive reaction. The von Frey filament provided 3 positive reactions in 5 tests with an interval of $10 \mathrm{sec}$ was used as PWT threshold.

Observation of spontaneous paw withdrawals: Rats were kept in cage and were allowed to move freely. Walking gait and the number of paw withdrawals within $90 \mathrm{sec}$ were observed.

Statistical analysis. SPSS 19.0 (SPSS Inc., Chicago, IL, USA) was used for all statistical analysis. Measurement data were expressed as mean $\pm \mathrm{SD}$, and comparison among multiple groups were performed using ANOVA and the post hoc was Dunnett test. $\mathrm{P}<0.05$ was considered to indicate a statistically significant difference.

\section{Results}

General information. All the selected rats were healthy female rats with mild temper. All rats were fed with LAD0011 feed. Model construction on all 150 rats was performed at the same time, and model was successfully constructed in 124 rats. All 124 rats showed no significant changes in weight and length after model construction $(\mathrm{P}<0.05)$. The average age of rats was $8.5 \pm 0.2$ weeks. The nutritional level was good and the average glucose concentration was $86.2 \pm 11.6$ (Table I). 


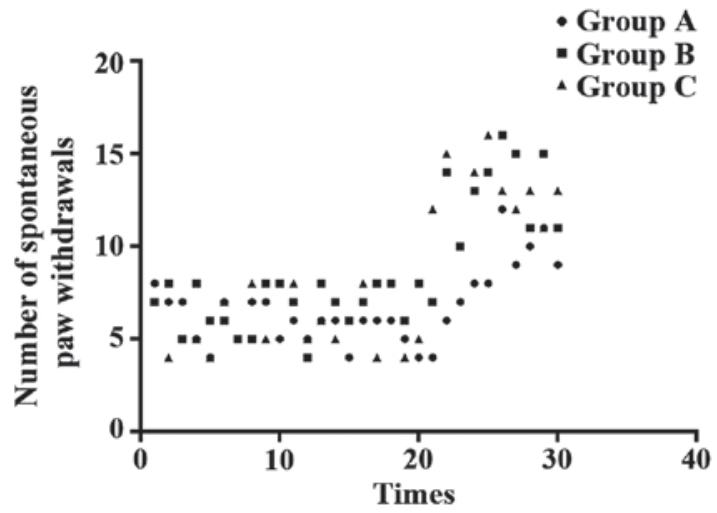

Figure 2. Observation of the number of spontaneous paw withdrawals in three groups of rats. The numbers of spontaneous paw withdrawals in control group were counted 3 times, and the average value was calculated to serve as reference $($ mean $=24.1)$. Increased numbers of spontaneous paw withdrawals were defined as effective treatment.

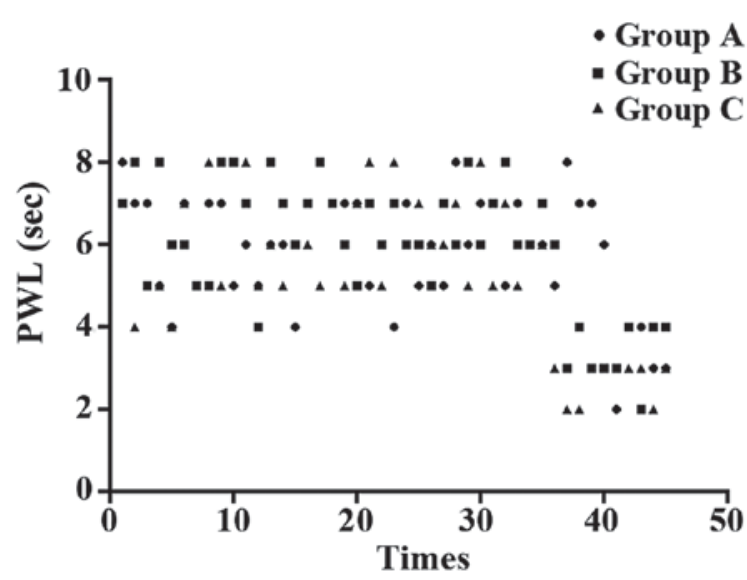

Figure 3. Observation of PWL in three groups of rats. PWL in control group were counted 3 times, and the average value was calculated to serve as reference $($ mean $=3.6$ ). Improved PWL was defined as effective treatment.

Treatment effect. The mean value of the rats in the control group was set as standard (spontaneous paw withdrawals 8.3 times, PWL $4.6 \mathrm{sec}$, PWT $15.7 \mathrm{~g}$ ), The improvement of pain behavior in rats was used to evaluate the effect of drug treatment. Twenty-five rats in group A showed different degrees of improvements, and 5 rats showed no improvement, and the effective rate was $83.3 \%$; 21 rats in group B showed different degrees of improvements, and 9 rats showed no improvement, and the effective rate was $70 \% ; 20$ rats in group $\mathrm{C}$ showed different degrees of improvements, and 10 rats showed no improvement, and the effective rate was $66.7 \%$. Significant differences in effective rate were found among the 3 groups, A showed highest effective rate $(\mathrm{P}<0.05)$, but no significant differences were found between group $\mathrm{B}$ and $\mathrm{C}(\mathrm{P}>0.05)$ (Fig. 1). Pain behavioral observation is shown in Figs. 2-4.

Comparison of physical improvement. With mean value of rats in control group as a standard (struggle domain area $7348.6 \mathrm{mv} x \mathrm{sec}$, cumulative immobility time $103.6 \mathrm{sec}$, single cumulative immobility time $60.5 \mathrm{sec}$ ), physical condition of rats in other groups were evaluated. Out of 30 rats in group A, 15 showed physical improvement, and the improvement rate

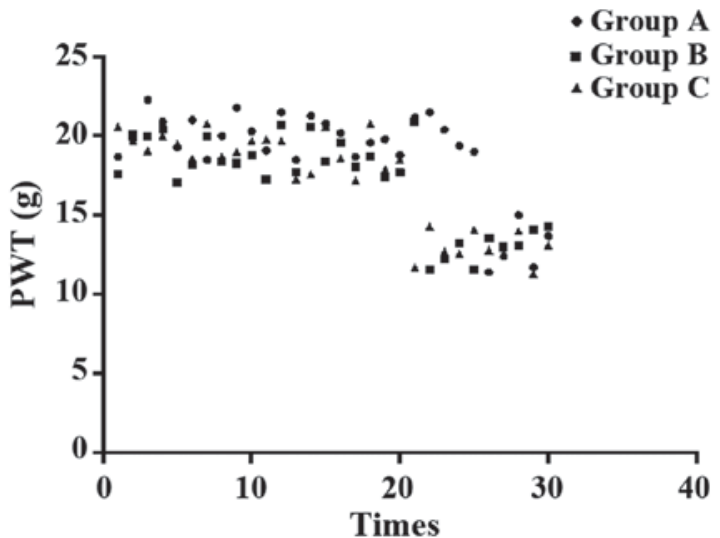

Figure 4. Observation of PWT in three groups of rats. PWT in control group were counted 3 times, and the average value was calculated to serve as reference $($ mean $=3.2)$. Improved PWT was defined as effective treatment.

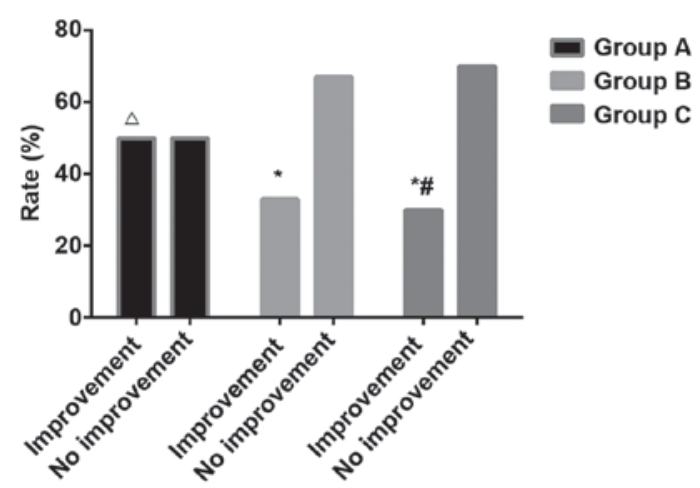

Figure 5. Physical improvement in three groups of rats. Physical improvement was observed in 15 out of 30 rats in group $\mathrm{A}$, and the improvement rate was $50 \%$; 10 out of 30 rats in group B showed physical improvement, and the improvement rate was $33.3 \%$; 9 out of 30 rats in group $\mathrm{C}$ showed physical improvement, and the improvement rate was $30.0 \%$. Significant differences were found among three groups, and group A showed the highest improvement rate $\left({ }^{\circ} \mathrm{P}=0.01\right.$, compared to groups $\mathrm{B}$ and $\left.\mathrm{C}\right)$. No significant differences were found between groups $\mathrm{B}$ and $\mathrm{C}\left({ }^{* \#} \mathrm{P}=0.0672\right)$.

was 50\%; 10 out of 30 rats in group B showed physical improvement, and the improvement rate was $33.3 \%$; 9 out of 30 rats in group $\mathrm{C}$ showed physical improvement, and the improvement rate was $30 \%$. Significant differences in improvement rate were found among the 3 groups, and group A showed greatest improvement rate $(\mathrm{P}<0.05)$ (Fig. 5). Results of the modified TST are shown in Figs. 6-8.

\section{Discussion}

Lung cancer is a major malignancy that affects human health. The incidence of lung cancer increases with age, and occurrence in individuals aged 55 or above is most common (12). Lung cancer is the third malignancy in the United States and is also a leading cause of death in patients (13). Smoking is a very important risk factor, and $70-90 \%$ of individuals with lung cancer in the United States smoke. Although smoking rates have been reduced, 30-40\% of individuals have a history of smoking (14). Therefore, it is imperative to improve the treatment of lung cancer, so as to improve the quality of life and survival of those patients. 


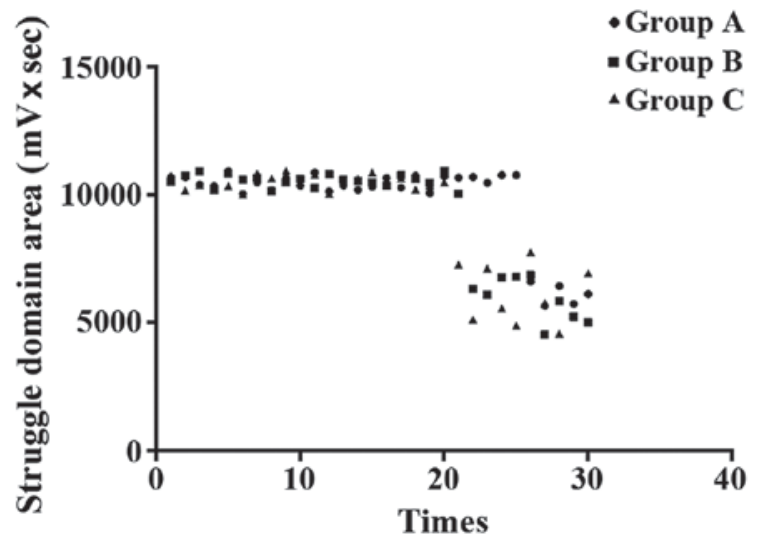

Figure 6. Observation of struggle domain area in three groups of rats. Struggle domain area in control group was counted 3 times, and the average value was calculated to serve as reference (mean $=6032 \mathrm{mV} \mathrm{x} \mathrm{sec})$. Improved struggle domain area was defined as effective treatment.

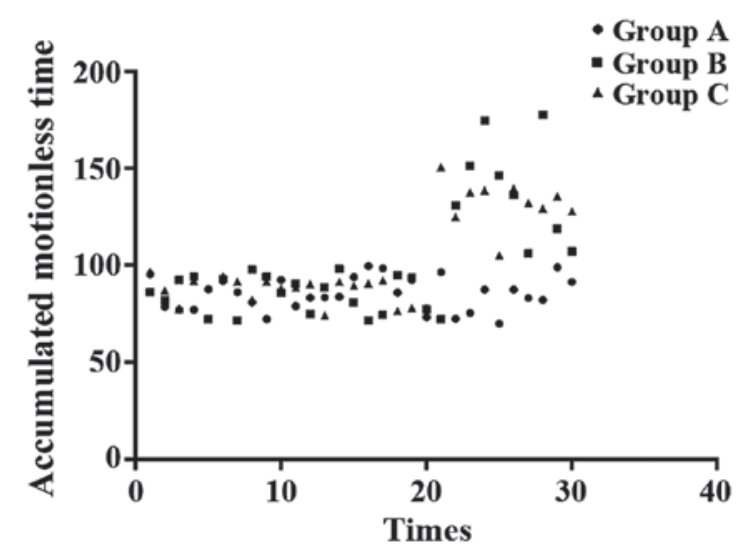

Figure 7. Observation of accumulated motionless time in three groups of rats. Accumulated motionless time in control group was counted 3 times, and the average value was calculated to serve as reference (mean $=84.5 \mathrm{sec}$ ) Improved accumulated motionless time was defined as effective treatment.

Bone is a major site of lung cancer metastasis, and $~ 40 \%$ of patients with non-small cell lung cancer (NSCLC) showed bone metastases during the course of disease, and bone tissue lesions were observed in more than $55 \%$ of newly diagnosed lung cancer patients. In this study, a lung cancer bone metastasis model was established to explore the therapeutic effect of zoledronic acid and ibandronate. The results of this study show that, compared with the pain behavior of control group, treatment with zoledronic acid improved spontaneous paw withdrawal, PWL and PWT in 21 out of 30 rats, and the treatment efficiency is $70 \%$; of the 30 rats treated with ibandronate, 20 showed improvement, and the effective rate was $66.7 \%$; of the 30 rats treated with both, the effective rate reached $83.3 \%$. No significant differences in the effective rate were found between the treatments with the two drugs alone $(\mathrm{P}>0.05)$. The above results showed that zoledronic acid combined with ibandronate can effectively improve the symptoms of lung cancer, and relieve cancer pain in rats. Zoledronic acid is the most commonly used bisphosphonic acid in the treatment of bone metastases (5). However, Barrett-Lee et al (15) found that zoledronic acid was superior to ibandronic acid in preventing bone-related events caused by bone metastases.

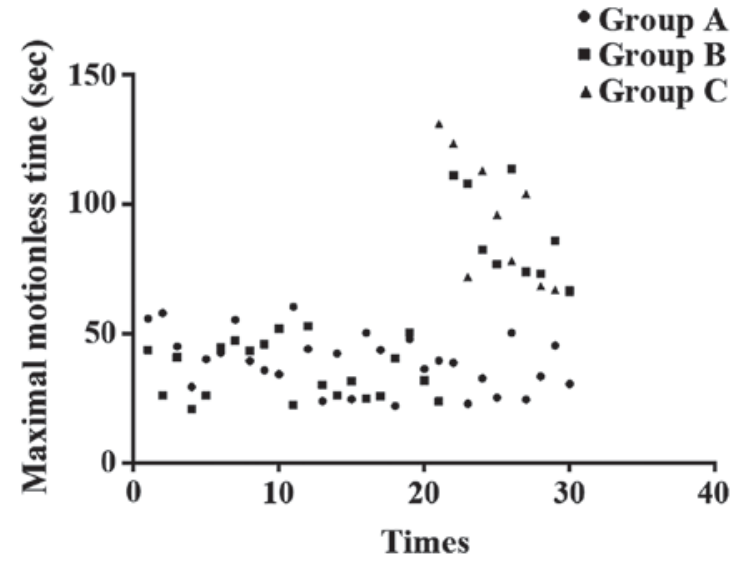

Figure 8. Observation of maximal motionless time in three groups of rats. Maximal motionless time in control group was counted 3 times, and the average value was calculated to serve as reference (mean $=42.5 \mathrm{sec}$ ). Improved maximal motionless time was defined as effective treatment.

This may be explained by the different dose, manner of administration, as well as duration of treatment used in our study. It has been suggested (16) that zoledronic acid can be used in the treatment of NSCLC to prevent or delay the occurrence of bone-related events. Previous findings have shown that, the therapeutic effect of zoledronic acid on lung cancer is achieved by inhibiting protein prenylation (17). Silva et al (18) indicated in their study that zoledronic acid could alter bone microenvironment and affect the growth of bone and bone tumors, so as to effectively reduce the risk of bone-related events. Ibandronate can also effectively reduce the risk of bone-related events in patients with lung cancer, and it has been reported that ibandronate can inhibit tumor cell adhesion, invasion, proliferation, and the interactions with bone matrix metalloproteinases (19).

Although zoledronic acid and ibandronate have good therapeutic effects on lung cancer, the combined use of zoledronic acid and ibandronate in the treatment of lung cancer bone metastases still has not been reported. Our study provided a reference for follow-up studies. Luedders et al found the combined use of zoledronic acid and ibandronate showed good tolerance and safety on renal toxicity in the 6-month treatment of breast cancer patients with bone metastases (20). Zoledronic acid and ibandronate acid are both bisphosphonates, and they can also be used in the treatment of osteoporosis $(21,22)$ and other bone diseases. More studies are needed to explore the roles of bisphosphonates in the treatment of bone diseases.

In summary, ibandronate and zoledronic acid have promising effects on improving the symptoms of bone metastases in lung cancer, and the combined use of the drugs can significantly improve the efficacy.

\section{Acknowledgements}

Not applicable.

\section{Funding}

No funding was received. 


\section{Availability of data and materials}

The datasets used and/or analyzed during the presend study are available from the corresponding author on reasonable request.

\section{Authors' contributions}

GW and JC established rat model. RM helped with radioisotope scanning. WX and CY recorded and analyzed pain behavior. $\mathrm{CN}$ observed spontaneous paw withdrawals. All authors read and approved the final manuscript.

\section{Ethics approval and consent to participate}

This study was approved by the Ethics Committee of Wuwei People's Hospital (Wuwei, China).

\section{Consent for publication}

Not applicable.

\section{Competing interests}

The authors declare that they have no competing interests.

\section{References}

1. Lortet-Tieulent J, Soerjomataram I, Ferlay J, Rutherford M, Weiderpass E and Bray F: International trends in lung cancer incidence by histological subtype: Adenocarcinoma stabilizing in men but still increasing in women. Lung Cancer 84: 13-22, 2014.

2. Tissot C, Couraud S, Tanguy R, Bringuier PP, Girard N and Souquet PJ: Clinical characteristics and outcome of patients with lung cancer harboring BRAF mutations. Lung Cancer 91: 23-28, 2016.

3. Borghaei H, Paz-Ares L, Horn L, Spigel DR, Steins M, Ready NE, Chow LQ, Vokes EE, Felip E, Holgado E, et al: Nivolumab versus docetaxel in advanced nonsquamous non-small-cell lung cancer. N Engl J Med 373: 1627-1639, 2015.

4. Reck M, Rodríguez-Abreu D, Robinson AG, Hui R, Csôszi T, Fülöp A, Gottfried M, Peled N, Tafreshi A, Cuffe S, et al; KEYNOTE-024 Investigators: Pembrolizumab versus chemotherapy for PD-L1-positive non-small-cell lung cancer. N Engl J Med 375: 1823-1833, 2016.

5. D'Antonio C, Passaro A, Gori B, Del Signore E, Migliorino MR, Ricciardi S, Fulvi A and de Marinis F: Bone and brain metastasis in lung cancer: Recent advances in therapeutic strategies. Ther Adv Med Oncol 6: 101-114, 2014.

6. Jung RS, Mittal BR, Santhosh S, Sood A, Bhattacharya A and Kapoor R: Is femoral uptake of Tc99m-methylene diphosphonate on bone scintigraphy in bronchogenic carcinoma an alarming sign: A case report and brief review of literature? Lung India 31: 280-281, 2014.

7. Henry D, Vadhan-Raj S, Hirsh V, von Moos R, Hungria V, Costa L, Woll PJ, Scagliotti G, Smith G, Feng A, et al: Delaying skeletal-related events in a randomized phase 3 study of denosumab versus zoledronic acid in patients with advanced cancer: An analysis of data from patients with solid tumors. Support Care Cancer 22: 679-687, 2014.

8. Murakami H, Yamanaka T, Seto T, Sugio K, Okamoto I, Sawa T, Hirashima T, Takeda K, Atagi S, Fukuoka M, et al: Phase II study of zoledronic acid combined with docetaxel for non-smallcell lung cancer: West Japan Oncology Group. Cancer Sci 105: 989-995, 2014.
9. Dimitroulis IA, Dervas A, Vasileiou S and Toumbis M: Comparative efficacy of ibandronic acid vs. Zoledronic acid in lung cancer patients with skeletal metastases. Do they alleviate the patient? J Thorac Oncol 9: S203-S204, 2014.

10. LeVasseur N, Clemons M, Hutton B, Shorr R and Jacobs C: Bone-targeted therapy use in patients with bone metastases from lung cancer: A systematic review of randomized controlled trials. Cancer Treat Rev 50: 183-193, 2016.

11. Miller RE, Jones JC, Tometsko M, Blake ML and Dougall WC: RANKL inhibition blocks osteolytic lesions and reduces skeletal tumor burden in models of non-small-cell lung cancer bone metastases. J Thorac Oncol 9: 345-354, 2014.

12. Moyer VA; U.S. Preventive Services Task Force: Screening for lung cancer: U.S. Preventive Services Task Force recommendation statement. Ann Intern Med 160: 330-338, 2014.

13. Moolgavkar SH, Holford TR, Levy DT, Kong CY, Foy M, Clarke L, Jeon J, Hazelton WD, Meza R, Schultz F, et al: Impact of reduced tobacco smoking on lung cancer mortality in the United States during 1975-2000. J Natl Cancer Inst 104: 541-548, 2012.

14. Humphrey L, Deffebach M, Pappas M, Baumann C, Artis K, Mitchell JP, Zakher B, Fu R and Slatore C: Screening for Lung Cancer: Systematic Review to Update the U.S. Preventive Services Task Force Recommendation [Internet]. U.S. Preventive Services Task Force Evidence Syntheses, formerly Systematic Evidence Reviews 157: 706, 2013.

15. Barrett-Lee P, Casbard A, Abraham J, Hood K, Coleman R, Simmonds P, Timmins H, Wheatley D, Grieve R, Griffiths G, et al: Oral ibandronic acid versus intravenous zoledronic acid in treatment of bone metastases from breast cancer: A randomised, open label, non-inferiority phase 3 trial. Lancet Oncol 15: 114-122, 2014.

16. Hendriks LE, Hermans BC, van den Beuken-van Everdingen MH, Hochstenbag MM and Dingemans AM: Effect of bisphosphonates, denosumab, and radioisotopes on bone pain and quality of life in patients with non-small cell lung cancer and bone metastases: A systematic review. J Thorac Oncol 11: 155-173, 2016.

17. Xie F, Li P, Gong J, Zhang J and Ma J: The bisphosphonate zoledronic acid effectively targets lung cancer cells by inhibition of protein prenylation. Biochem Biophys Res Commun 467: 664-669, 2015.

18. Silva SC, Wilson C and Woll PJ: Bone-targeted agents in the treatment of lung cancer. Ther Adv Med Oncol 7: 219-228, 2015.

19. Liu J, Huang W, Zhou R, Jia S, Tang W, Luo Y and Zhang J: Bisphosphonates in the treatment of patients with metastatic breast, lung, and prostate cancer: A Meta-analysis. Medicine (Baltimore) 94: e2014, 2015.

20. Luedders DW, Steinhoff J, Thill M, Rody A and Bohlmann MK: Lack of difference in acute nephrotoxicity of intravenous bisphosphonates zoledronic acid and ibandronate in women with breast cancer and bone metastases. Anticancer Res 35: 1797-1802, 2015.

21. Qaseem A, Forciea MA, McLean RM and Denberg TD; Clinical Guidelines Committee of the American College of Physicians: Treatment of low bone density or osteoporosis to prevent fractures in men and women: A clinical practice guideline update from the American College of Physicians. Ann Intern Med 166: 818-839, 2017.

22. Jamil K, Zacharin M, Foster B and Munns CF: Protocol for a randomised control trial of bisphosphonate (zoledronic acid) treatment in childhood femoral head avascular necrosis due to Perthes disease. BMJ Paediatrics Open 1: e000084, 2017. http://bmjpaedsopen.bmj.com/content/1/1/e000084.

This work is licensed under a Creative Commons Attribution-NonCommercial-NoDerivatives 4.0 International (CC BY-NC-ND 4.0) License. 